\title{
Differential cross section for electron impact excitation of metastable helium measured by the atomic time-of-flight method $\dagger$
}

\author{
Arthur G Zajonc, Gabriel Weinreich and Jens C Zorn \\ Randall Laboratory of Physics, University of Michigan, Ann Arbor, Michigan 48109, \\ USA
}

Received 20 October 1976

\begin{abstract}
We have measured the differential cross section for the inelastic process $\mathrm{He}\left(1^{1} \mathrm{~S}_{0}\right)+\mathrm{e}^{-} \rightarrow \mathrm{He}\left(2^{3} \mathrm{~S}_{1}\right)+\mathrm{e}^{-}$just above threshold by bombarding a beam of helium atoms with electrons from a pulsed electron gun and measuring the times of flight of the excited metastable atoms to a distant detector. Since each time of flight corresponds to a definite value of the electron scattering angle, the angular distribution of electrons scattered over the entire range $0-180^{\circ}$ is deduced from the data.
\end{abstract}

The excitation of the $2^{3} \mathrm{~S}_{1}$ state of helium by electron impact has a considerable history of both experimental and theoretical investigation. The spin flip necessary for singlet-triplet transitions demands that the interaction proceed at close range, while the high polarizability of metastable helium superimposes a long-range interaction between the scattered electron and atom. Coherent addition of these two factors leads to a great deal of structure in the cross section.

Experimental determinations of the differential cross section for this process have been made by several groups (Ehrhardt and Willmann 1967, Ehrhardt et al 1968, Andrick et al 1975, Pichou et al 1975, 1976), all of whom identify the reaction by measuring the energy and direction of the scattered electrons. Near threshold, such measurements place heavy demands on the electron optics and calibration procedures since electrons of very low energy are hard to handle. Moreover, apparatus constraints make it difficult to obtain data near $0^{\circ}$ and $180^{\circ}$. Thus it is useful to measure the cross sections by a method which avoids these difficulties by detecting the excited atoms rather than the scattered electrons.

In this experiment, an electron gun (resolution $0.25 \mathrm{eV}$ FWHM) operated in a pulsed mode ( $5 \mu \mathrm{s}$ pulse width, $5 \mathrm{kHz}$ repetition rate) excites some atoms in a well collimated helium beam to the metastable state. The axis of the gun is oriented at right angles to the incoming atoms. The excited (and deflected) atoms are detected with a windowless electron multiplier whose angular resolution is $0.5^{\circ}$. The distribution of times of flight of the atoms, from gun pulse to detection, constitutes our primary data. In an earlier experiment of this type, Heppner and Zorn (1974) demonstrated the striking effect of the differential cross section on the form of the metastable time-offlight (MTOF) spectra. In the present experiment we have improved the quality of the data and have used a more comprehensive method to analyse that data. As † Research supported in part by the National Science Foundation. 
a result, we now obtain values for the differential cross section over the entire angular range of $0-180^{\circ}$ directly from the MTOF spectra.

A theoretical description of the effects on MTOF spectra of recoil kinematics, ground-state velocity distribution and differential cross section is given by Pearl (1970) and Pearl et al (1976)†. The essential point necessary for extraction of the differential cross section is that to a given electron energy, detector angle and metastable atom speed there corresponds only one value of $\cos \theta$, where $\theta$ is the electron scattering angle. To see this, let $U_{0}$ and $U$ be, respectively, the magnitudes of the incoming and outgoing electron momenta; and let $V$ be the magnitude of the outgoing atom momentum. If $\alpha$ is the detector angle (that is, the angle by which the atom is deflected from its original direction), conservation of momentum in the direction of the incoming electron beam is embodied in the equation

$$
U_{0}=U \cos \theta+V \sin \alpha .
$$

Because the ratio of electron mass to atom mass is very small, the collision decreases the electron's kinetic energy by an amount equal to the metastable excitation energy of the atom, thus determining $U$. Hence, equation (1) can be solved for $\cos \theta$ in an unequivocal way.

The above 'ideal' situation is complicated somewhat by the fact that the apparatus resolution is not perfect, as a result of which the measured intensity at a given time of flight comes from a small but finite range of $\theta$. For this reason, we have extracted our cross section curves by an iterative procedure which involves comparison of the data with a computer-simulated spectrum that incorporates such factors as finite resolution of the electron gun, finite detector resolution, the ground-state velocity distribution and, finally, an assumed cross section which is initially set to be isotropic. By taking ratios of experimental to simulated count rates, we obtain the next trial form of the cross section. This procedure converges very rapidly-typically after two iterations.

In figure 1 we show an experimental MTOF spectrum along with the simulations obtained using an assumed s-wave cross section (broken curve) and using the cross section found from the ratio computation (full curve). The strong effect of the cross section on the form of the MTOF spectrum is evident. In figure 2 we show the cross section for the inelastic process as a function of electron scattering angle for a number of electron energies below the $20.62 \mathrm{eV}$ threshold of the $2^{1} \mathrm{~S}_{0}$ state. From that figure one sees that the present experiment covers a broader angular range than do the other measurements; further, it provides values for the cross section as an almost continuous function of angle over that range.

Ehrhardt and Willmann (1967) interpreted their data as indicating that the electron scattering below $19.95 \mathrm{eV}$ is essentially s-wave, and this appeared to be supported by the early MTOF results of Heppner and Zorn (1974). The larger angular range and increased resolution now available in both conventional scattering experiments (Andrick et al 1975, Pichou et al 1975, 1976) and in the present MTOF work shows this not to be so; there is considerable anisotropy in the electron distribution even a few millivolts above threshold. Nesbet (1975) and Oberoi and Nesbet (1973) have studied the near-threshold region theoretically by a variational method; they conclude that while the s-wave reaches a maximum near $19.89 \mathrm{eV}$, the p-component at that energy is already $30 \%$ of the s-component. Thus interference should be apparent, in agreement with what we observe.

$\dagger$ Pearl J C, Weinreich G and Zorn J C in preparation. 


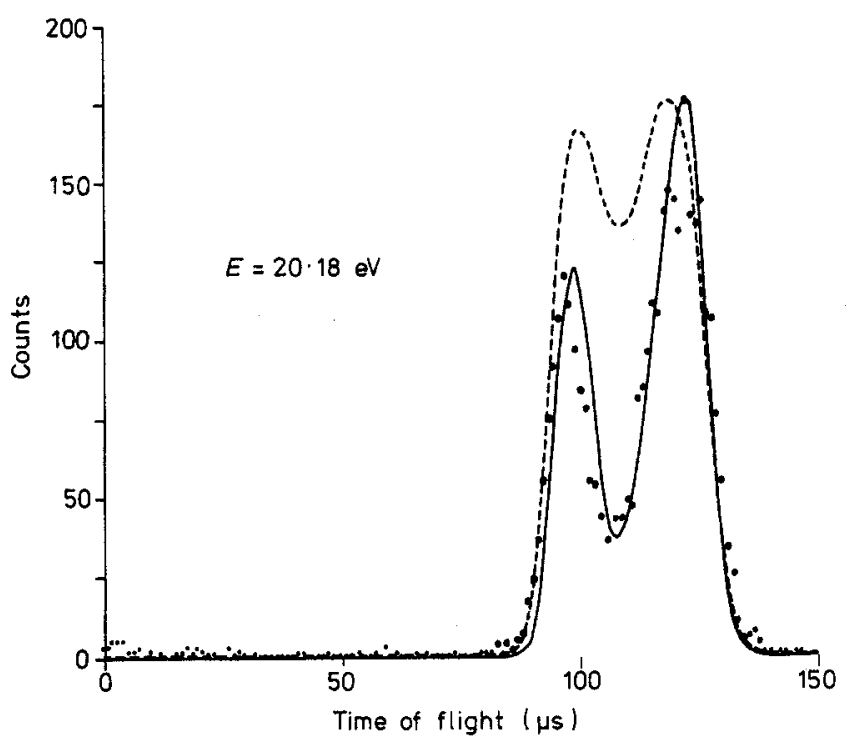

Figure 1. Comparison of a typical experimental MTOF spectrum (dots) with simulated spectra. From this single run of $2000 \mathrm{~s}$ duration we can obtain the $20.18 \mathrm{eV}$ differential cross section curve shown in figure 2 ; that cross section in the simulation program yields the full curve in this figure. A simulation based on an isotropic cross section is shown (broken curve) for comparison.

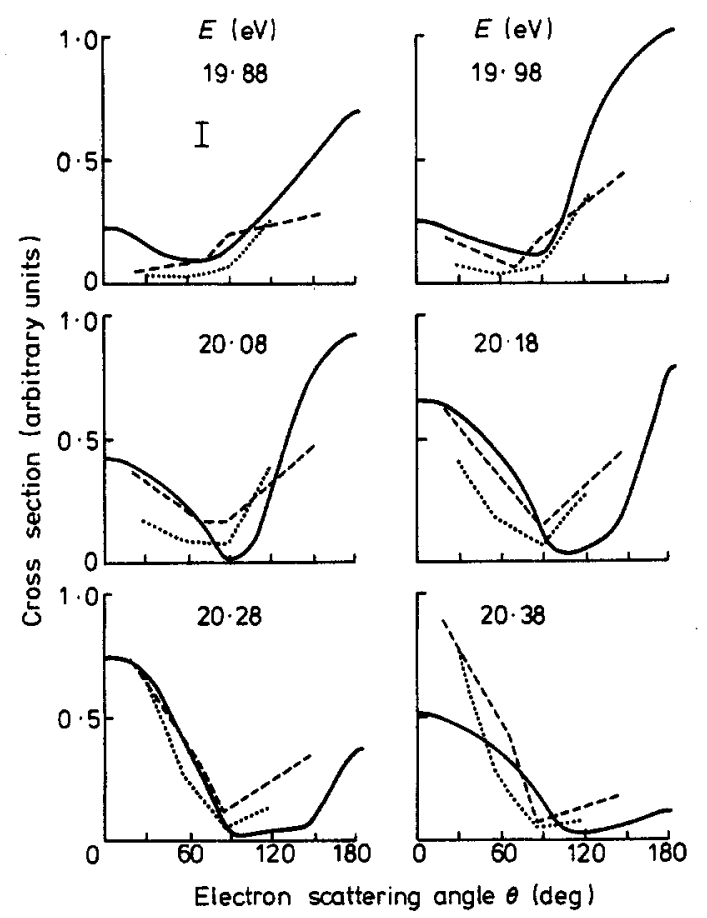

Figure 2. Differential cross section for excitation of $2^{3} \mathrm{~S}_{1}$ helium near threshold. Our results (full curves with a typical error bar shown on the $19.88 \mathrm{eV}$ plot) are compared with those of Pichou et al (dotted lines connecting their data points) and those of Andrick et al (broken lines). The values of all experiments are normalized to be the same at $30^{\circ}$ for an energy of $20.28 \mathrm{eV}$. 
The total cross section for production of the $2^{3} \mathrm{~S}_{1}$ state by electron impact shows a peak near $20.4 \mathrm{eV}$ that has been interpreted as a p-wave resonance, and the location of that resonance is of some interest. The close-coupling calculations by Burke et al (1969) and the variational calculation by Oberoi and Nesbet (1973) both predict the resonance to be in the region $20 \cdot 17-20 \cdot 20 \mathrm{eV}$. Since our data covers the complete angular range it is easy to pick out the energy at which the scattering is most ' $p$-like' in the sense of being equally peaked at $0^{\circ}$ and $180^{\circ}$, with a minimum at $90^{\circ}$. From the results shown in figure 2 this energy is seen to be just above $20.18 \mathrm{eV}$, but higher partial waves are also evident even at these relatively low energies. Recent electronscattering data has been analysed in terms of a partial-wave expansion by Linder and his colleagues ( $F$ Linder 1976 private communication), and they find the $p$-wave maximum to be at $20 \cdot 25 \mathrm{eV}$, somewhat above the value suggested by our data.

A complete report is in preparation. We acknowledge helpful conversations with F Linder, R K Nesbet, and R A Heppner, and friendly assistance from M Marafi and $\mathrm{E}$ Arnold. We thank D A Crosby for his numerous contributions to these experiments.

\section{References}

Andrick D. Langhans L, Linder F and Seng G 1975 Proc. 9th Int. Conf. on Physics of Electronic and Atomic Collisions ed J S Risley and R Geballe (Seattle: University of Washington Press) Abstracts pp 833-4

Burke P G, Cooper J W and Ormonde S 1969 Phys. Rev. 183245

Ehrhardt H, Langhans L and Linder F 1968 Z. Phys. 214179

Ehrhardt H and Willmann K 1967 Z. Phys. 2031

Heppner R A and Zorn J C 1974 Phys. Rev. Lett. 331321

Nesbet R K 1975 Phys. Rev. A 12444

Oberoi R S and Nesbet R K 1973 Phys. Rev. A 82969

Pearl J C 1970 PhD Thesis University of Michigan (Ann Arbor, Michigan: University Microfilms)

Pichou F, Huetz A, Joyez G, Landau M and Mazeau J 1975 J. Phys. B: Atom. Molec. Phys. 8 L236

1976 J. Phys. B: Atom. Molec. Phys. 9933 